http://jmscr.igmpublication.org/home/ ISSN (e)-2347-176x ISSN (p) 2455-0450 crossref DOI: https://dx.doi.org/10.18535/jmscr/v9i5.27

\title{
The Diagnostic Potential of CB NAAT in Detecting Drug Resistance in Smear-Negative Pulmonary Tuberculosis Patients: A Retrospective Study
}

\author{
Authors \\ Dr Nagaraja B S $\mathbf{S}^{1}$, Dr Deepak K $\mathbf{J}^{2}$
}

${ }^{1}$ Professor, Department of General Medicine, Bangalore Medical College and Research Center Bangalore

${ }^{2}$ Post Graduate in General Medicine, Bangalore Medical College and Research Institute Bangalore

\begin{abstract}
Background: There is paucity of data regarding the diagnostic role of cartridge-based nucleic acid amplification test (CBNAAT), in the diagnosis of drug resistant sputum smear-negative PTB cases.

Aim: To determine the diagnostic role of CBNAAT in detecting drug resistance smear negative PTB.

Materials \& Methods: The retrospective study obtained the data on patient samples registered to Revised National TB Control Programme during the period January 2018 to December 2019 due to suspected PTB. Acid-Fast Bacilli (AFB) smear and CBNAAT were carried out for all the collected samples. The incidence of rifampicin resistance was also evaluated. Descriptive statistics was carried out using suitable parametric and non-parametric tests of significance. Comparison of parameters was carried out using chi-square test for counts data.

Results: A total 3652 suspected cases were screened using AFB smear and CBNAAT, and the corresponding number of positive cases reported were 291 (7.97\%) and 337(9.23\%) (P 0.055, Table 1, Fig. 1). The mean age of the positive patients was $41.53 \pm 15.22$ years and the male-to-female ratio noted was 1:0.33. Out of 337 positive patients, 46(13.65\%) were diagnosed as negative in AFB, and all these patients were diagnosed as positive (100\%) in CBNAAT (Table 2, Fig. 2,P < 0.00001). Thirty-five (10.39\%) TB-positive patients were found to be drug resistant $(P<0.00001)$ and the number of subjects resistant to rifampicin, isoniazid and both were $31(9.2 \%), 3(0.89 \%)$ and $1(0.3 \%)$ respectively (Table 3, Fig. 3). In smear negative patients $(n=46), 4$ were found rifampicin resistant $(8.70 \%)$, whereas in smear-positive patients $(n=31,10.65 \%)$, resistance to rifampicin, isoniazid and both were noted in $27(9.28 \%), 3(1.03 \%)$ and $1(0.34 \%)$ subjects respectively (Fig. 4 ).

Conclusion: The study corroborates the potential of CBNAAT in identifying drug resistance in smear negative PTB.
\end{abstract}

\section{Introduction}

Tuberculosis which is caused by bacteria of the mycobacterium tuberculosis complex is one of the oldest diseases known to affect humans and the top cause of infectious death worldwide. This disease most often affects the lungs although other organs are involved in up to $1 / 3 \mathrm{rd}$ of cases. If properly treated TB caused by drug susceptible strains is curable in the vast majority of cases. if untreated the disease may be fatal within 5 years in $50-65 \%$ of cases. Transmission usually takes place through airborne spread of droplet nucliei produced by patients with infectious $\mathrm{ptb}^{1}$. 
The key to the early diagnosis of tb is the high index of suspicion. Diagnosis is not difficult in persons belonging to high risk populations who present with typical symptoms and a classic chest radiograph showing upper lobe infiltrates with cavities. On the other hand the diagnosis can be easily missed in an elderly person or a teenager with a focal infiltrate. ${ }^{1}$

In many low and middle income settings a presumptive diagnosis is still commonly based on the finding of afb on microscopic examination of a diagnostic specimen such as a smear of expectorated sputum or of tissue. Although inexpensive, AFB microscopy has relatively low sensitivity (40-60\%)in culture confirmed cases of ptb. Several test systems based on amplification of micobacterial nucleic acid have become available in the past few years and are now the preferred first line diagnostic tests. These tests are progressively replacing smear microscopy as they ensure rapid confirmation of all types of TB. ${ }^{1}$

Pulmonary tuberculosis is still one of the commonest cause of infectious disease related morbidity and mortality in the developing countries. $^{2}$

Cartidge based nucleic acid amplification test (CBNAAT) is a nested polymerase chain reaction (PCR) technique that identifies small quantities of genetic elements of mycobacterium tuberculosis from clinical specimens and it can identify resistance to rifampicin, the surrogate marker of multi-drug resistant (MDR) tuberculosis, at the same time. CBNAAT is completely automated, has minimal biosafety hazard and can give result within two hours. World health organization has endorsed the use of this rapid molecular diagnostic test for diagnosis of tuberculosis with special emphasis on drug-resistant tuberculosis, human immunodeficiency virus (HIV) AND TB co-infection, paediatric tuberculosis, extrapulmonary tuberculosis and smear negative pulmonary tuberculosis.. ${ }^{3,4}$

There has been paucity of regarding diagnostic role of CBNAAT specially in diagnosis of sputum smear-negative PTB cases. In this background, the present study was carried out to determine the diagnostic role of CBNAAT in detecting drug resistance in smear negative $\mathrm{PTB}$.

\section{Aims and objectives of the study}

A. To determine the occurrence of smear negative CBNAAT positive pulmonary TB cases.

B. To determine the occurrence of rifampicin resistance in such cases.

\section{Materials and Methods}

Source of Data: study was conducted based on the records available in Bowring and Lady Curzon Hospital Attached to BMCRI during the Period of January 2018 to December 2019 retrospectively. All cases of pulmonary tuberculosis during the specified period were enrolled for the study

\section{Inclusion Criteria}

1. Patients above 18 years of age.

2. Patients who were subjected to CBNAAT after being smear negative.

\section{Exclusion Criteria}

1. Patients less than 18 years of age

2. Smear positive PTB

3. Extra pulmonary TB

After obtaining ethical clearance and approval from the institutional ethics committee of BMCRI, the data of individuals who were suspected to have PTB and subjected to sputum smear examination and found positive as well as negative and who were negative for such sputum smear and being subjected to CBNAAT studies in due course of time and found positive for $\mathrm{CB}$ NAAT were obtained from the RNTCP registry of Bowring And Lady Curzon Hospital attached to BMCRI. The same was analysed and the incidence of rifampicin resistance in such cases was studied. Descriptive statistics was carried out using suitable parametric and non- parametric tests of significance. Comparison of parameters was carried out using chi-square test for counts data. 
Suspected PTB Individuals

sputum smear examination N3652

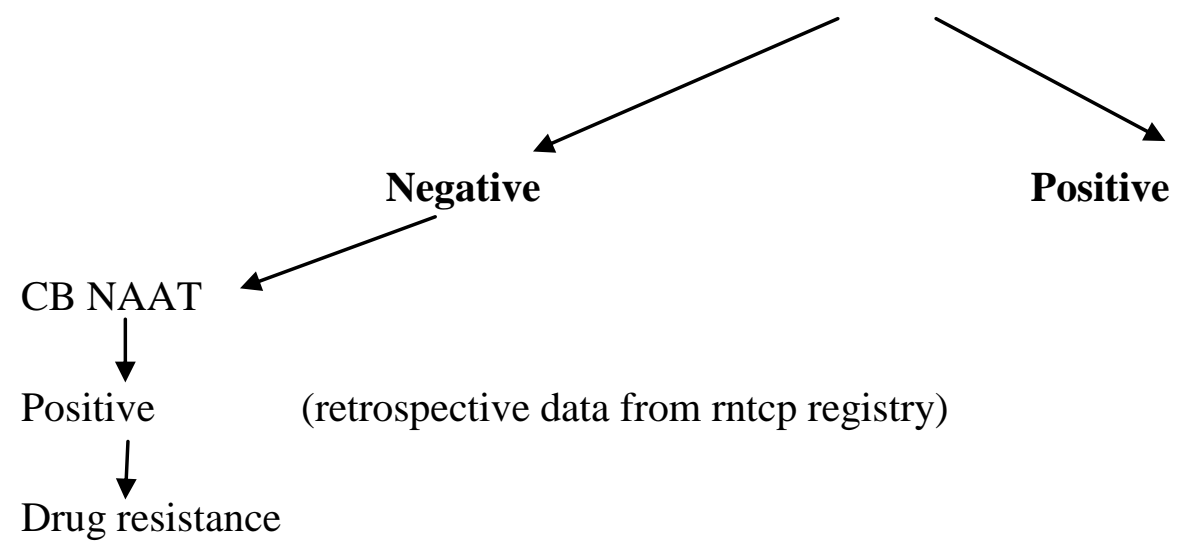

\section{Results}

A total 3652 suspected cases were screened using AFB smear and CBNAAT, and the corresponding number of positive cases reported were 291 $(7.97 \%)$ and 337(9.23\%) (p 0.055, table 1, fig. 1). The mean age of the positive patients was $41.53 \pm 15.22$ years and the male-to-female ratio noted was 1:0.33. Out of 337 positive patients, 46(13.65\%) were diagnosed as negative in AFB, and all these patients were diagnosed as positive $(100 \%)$ in CBNAAT (table 2, fig. 2,p < 0.00001). thirty-five $(10.39 \%)$ tb-positive patients were found to be drug resistant ( $\mathrm{p}<0.00001)$ and the number of subjects resistant to rifampicin, isoniazid and both were $31(9.2 \%), 3(0.89 \%)$ and
$1(0.3 \%)$ respectively (table 3 , fig. 3$)$. In smear negative patients $(n=46), 4$ were found rifampicin resistant $(8.70 \%)$, whereas in smear-positive patients $(n=31,10.65 \%)$, resistance to rifampicin, isoniazid and both were noted in $27(9.28 \%)$, $3(1.03 \%)$ and $1(0.34 \%)$ subjects respectively (fig. 4).

Total 3652 tb suspected patients were screened for TB using AFB smear and CBNAAT methods. Number of positive patients in afb smear method was $291(7.97 \%)$ where as in CBNAAT it was $337(9.23 \%$ ) (table 1). The screening of TB patients using these two methods was found to be statistically significant at $91 \%$ confidence interval with p-value of 0.055 .

Table 1: comparison of sputum smear and cb-naat in screening tb positive patients

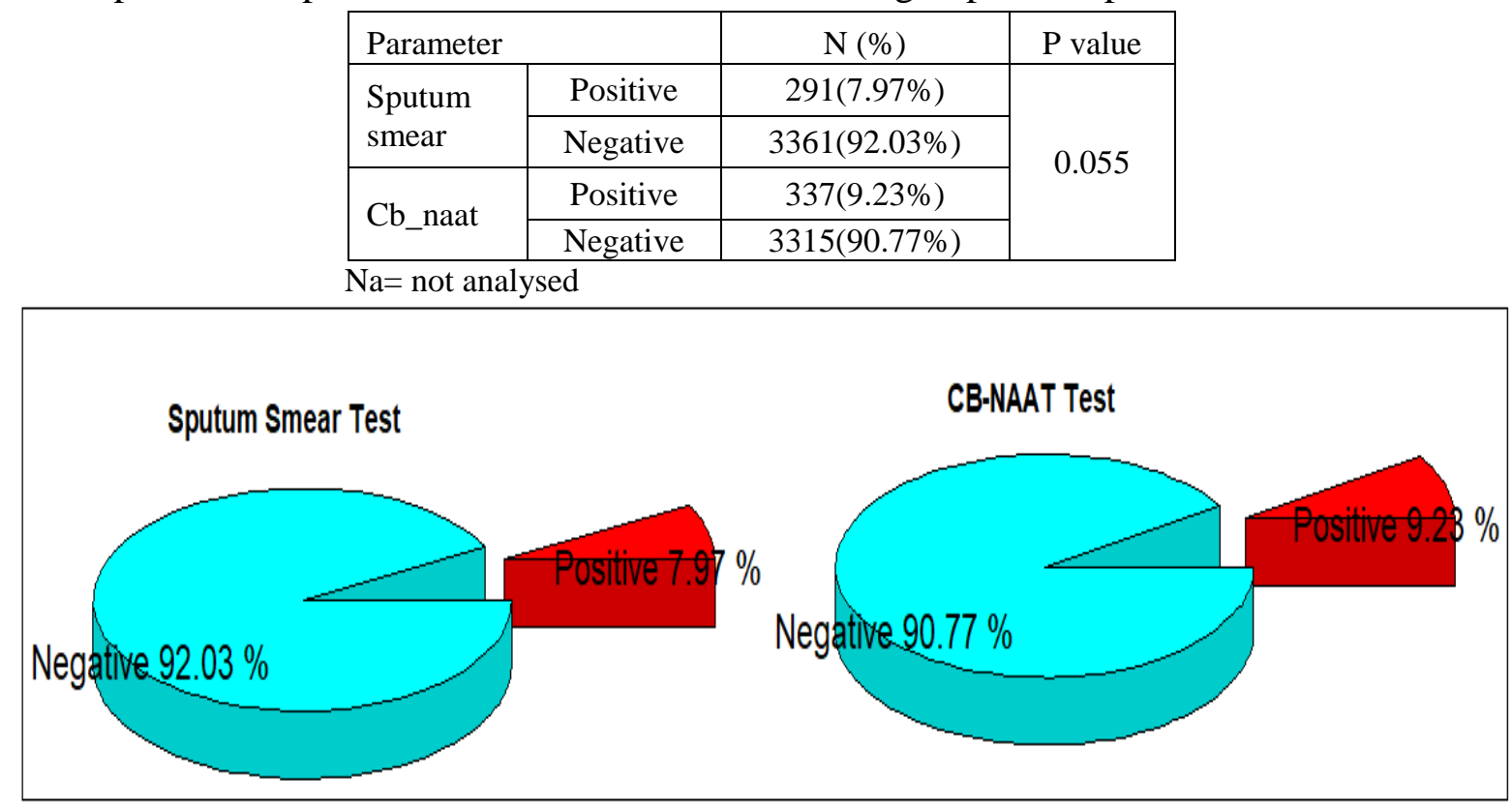

Figure 1: screening of tb positive patients using sputum smear and CB-NAAT 


\section{JMSCR Vol||09||Issue||05||Page 146-152||May}

Mean age of 337 positive patients was $41.53 \pm 15.22$ years. There were $253(75.07 \%)$ and 84(24.93\%) were male and female patients in the positive population. it was found that out of 337 positive patients $46(13.65 \%)$ were diagnosed as negative in AFB smear method were as all the patients were diagnosed as positive (100\%) positive in CBNAAT method (table 2). Difference in two methods in diagnosing tb was statistically significant at confidence interval $99 \%$ with $\mathrm{p}$ value $<0.00001$.

Table 2: Comparison of results of sputum smear and CB NAAT in positive patients $(n=337)$

\begin{tabular}{|c|c|c|c|}
\hline \multicolumn{2}{|c|}{ Parameter } & Mean \pm sd & $P$ value \\
\hline \multicolumn{2}{|c|}{ Age $($ mean \pm sd $)$} & $41.53 \pm 15.22$ & $\mathrm{Na}$ \\
\hline & & $\mathrm{N}(\%)$ & \\
\hline \multirow[t]{2}{*}{ Gender } & Male & $253(75.07 \%)$ & \multirow{2}{*}{$\mathrm{Na}$} \\
\hline & Female & $84(24.93 \%)$ & \\
\hline \multirow{2}{*}{$\begin{array}{l}\text { Sputum } \\
\text { smear }\end{array}$} & Positive & $291(86.35 \%)$ & \multirow{4}{*}{$<0.00001$} \\
\hline & Negative & $46(13.65 \%)$ & \\
\hline \multirow[t]{2}{*}{ Cb_naat } & Positive & $337(100 \%)$ & \\
\hline & Negative & 0 & \\
\hline
\end{tabular}

$\mathrm{Na}=$ not analysed

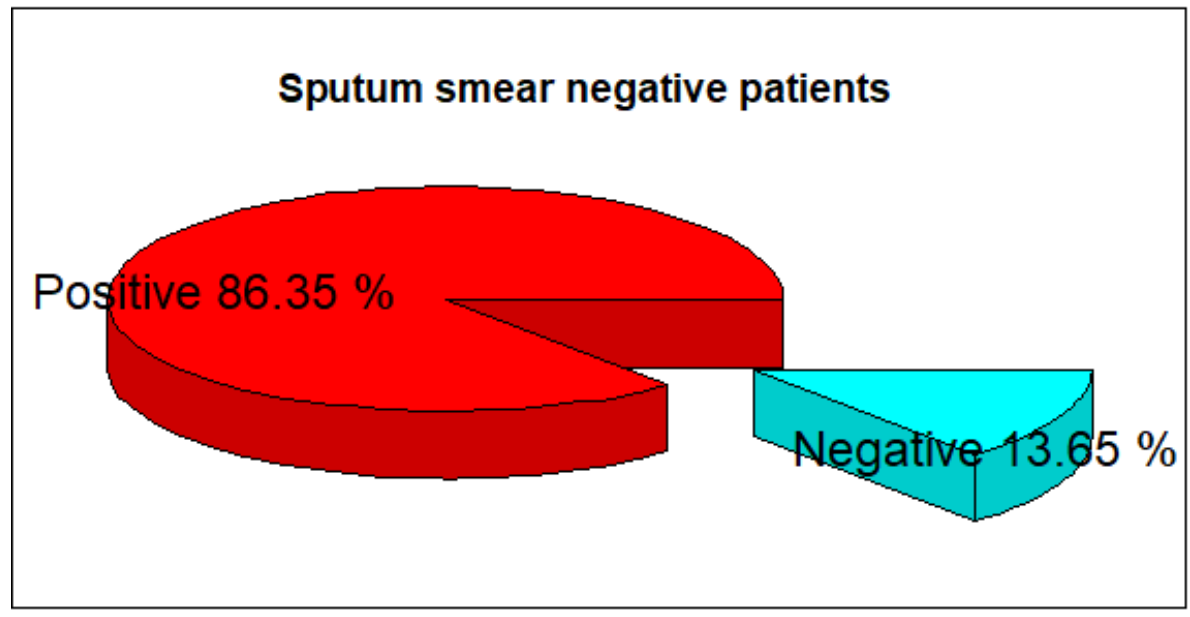

Figure 2: Sputum smear negative population in tb positive patients

Along with accuracy in diagnosing tb, CB NAAT also has the advantage of detecting drug resistant population. In the current study, 35(10.39\%) tb positive patients were found to be drug resistant. It was found that $31(9.20 \%), 3(0.89 \%)$ and $1(0.30 \%)$ were resistant to rifampicin (rif), isoniazid (inh) and both respectively (table 3 ). In smear negative patients $(n=46), 4$ patients were found rif resistant $(8.70 \%)$ whereas in smear positive total $31(10.65 \%)$ patients were found to be drug resistant in which $27(9.28 \%), 3(1.03 \%)$ and $1(0.34 \%)$ were resistant to rifampicin (rif), isoniazid (inh) and both respectively. It was shown that the detection of drug resistance in tb positive is statistically highly significant at $\mathrm{p}$ value $<0.00001$. 
Table 3: Resistance to drug in CB NAAT positive population

\begin{tabular}{|c|c|c|c|}
\hline Group & $\begin{array}{c}\text { Drug } \\
\text { resistant }\end{array}$ & Drug sensitive & P- value \\
\hline Total positive population $(\mathrm{n}=337)$ & $35(10.39 \%)$ & $302(89.61 \%)$ & $\mathrm{Na}$ \\
\hline Rif in cb_naatpositive population $(\mathrm{n}=337)$ & $31(9.20 \%)$ & $337((90.80 \%)$ & \multirow{3}{*}{$<0.00001$} \\
\hline Inh in positive population $(\mathrm{n}=337)$ & $3(0.89 \%)$ & $43(99.11 \%)$ & \\
\hline Inh+rif in positive population $(\mathrm{n}=337)$ & $1(0.30 \%)$ & $45(99.70 \%)$ & \\
\hline Rif in smear negative population $(\mathrm{n}=46)$ & $4(8.70 \%)$ & $42(91.30 \%)$ & \multirow{4}{*}{$<0.00001$} \\
\hline Rif in smear positive population $(\mathrm{n}=291)$ & $27(9.28 \%)$ & $264(90.72 \%)$ & \\
\hline Inh in smear positive population $(\mathrm{n}=291)$ & $3(1.03 \%)$ & $288(98.97 \%)$ & \\
\hline Inh+rif in smear positive population $(\mathrm{n}=291)$ & $1(0.34 \%)$ & $290(99.66 \%)$ & \\
\hline
\end{tabular}

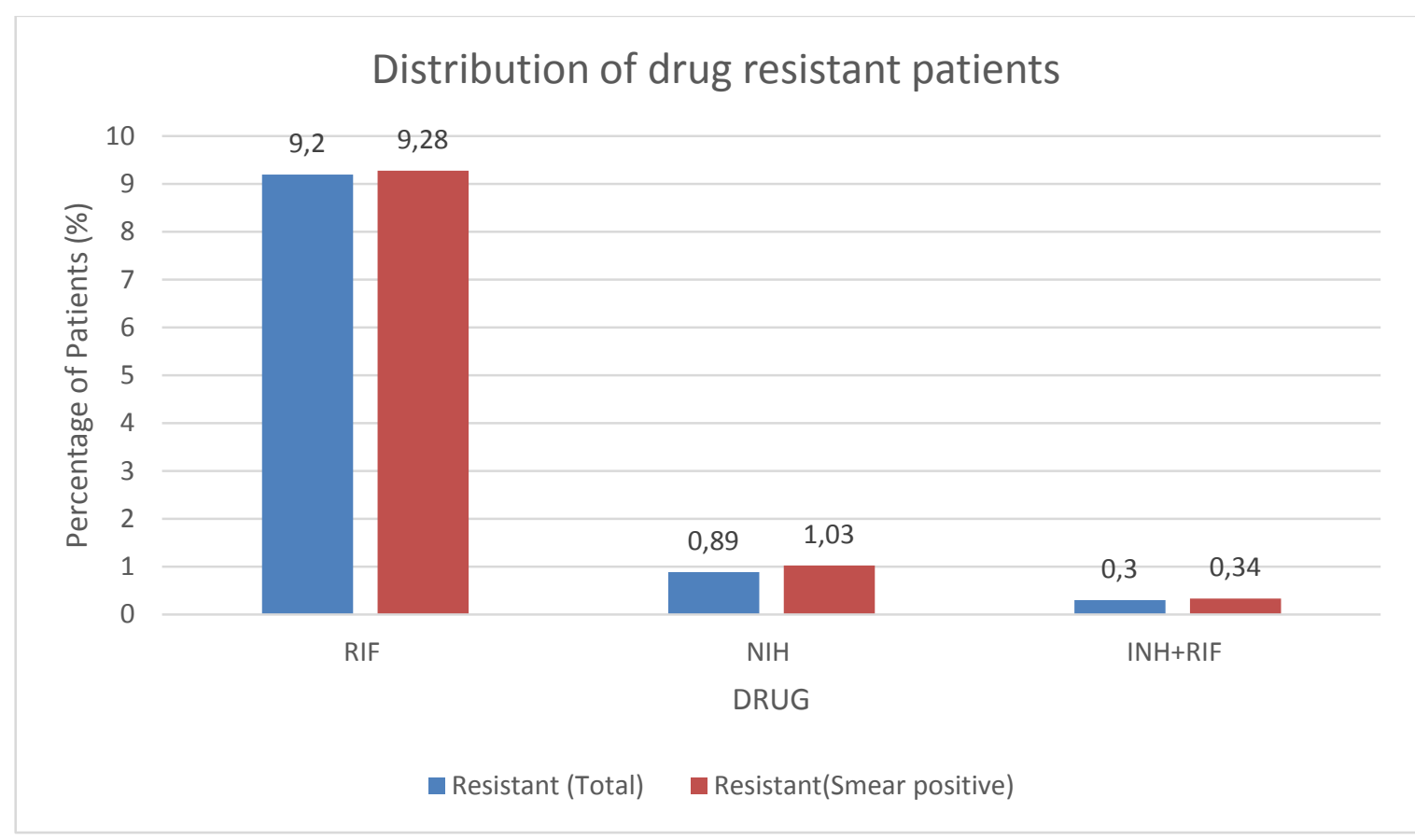

Figure 3: Drug Resistant percentage in total positive as well as smear positive patients

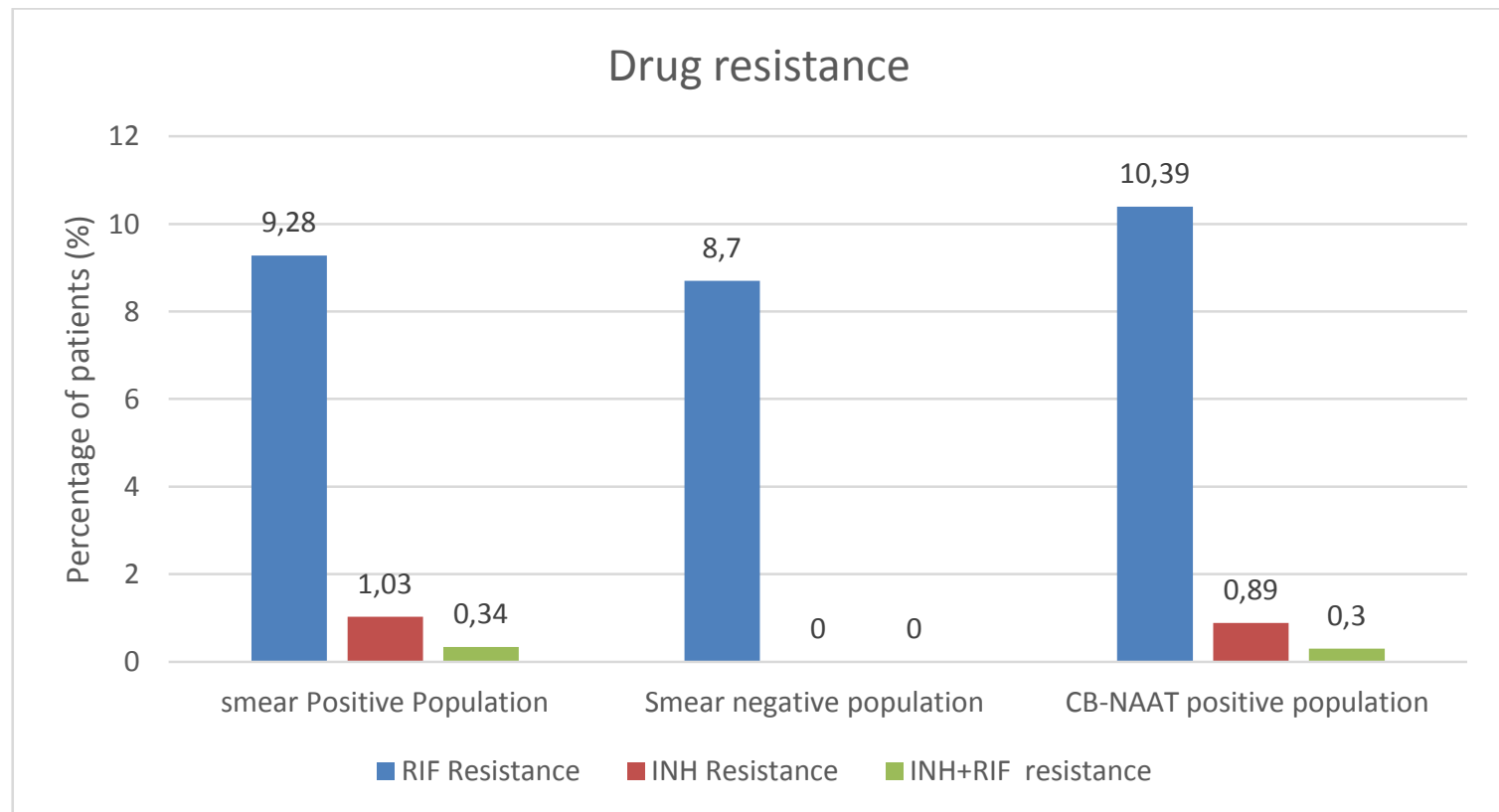

Figure 4: Drug Resistance in smear positive, negative as well as in CB-NAAT positive patients 
Table: 4 Characteristics of Patients with Smear Negative CB NAAT Positive Reports (N-46)

\begin{tabular}{|l|c|c|c|}
\hline Age group & No.of patients & Male & Female \\
\hline $18-30$ yrs & 12 & 06 & 06 \\
\hline $31-40$ yrs & 08 & 08 & 0 \\
\hline $41-50$ yrs & 11 & 09 & 02 \\
\hline $51-60$ yrs & 07 & 06 & 01 \\
\hline$>60$ yrs & 08 & 08 & 0 \\
\hline Total & 46 & $37(80.4 \%)$ & $09(19.6 \%)$ \\
\hline
\end{tabular}

12 patients $(26.1 \%)$ patients belonged to the age group of 18-30 years with the lowest age being 18 years and the highest being 83.mean age was 44.2. Predominantly found to be males $80.4 \%$.

The respective age of patients with rifampicin resistance in 4 patients was 19,25,43, and 60 years respectively and 3 out of 4 were males.

\section{$\underline{\text { Discussion }}$}

Study conducted By Subhasis Mukherjee et al showed that CBNAAT is a very useful and rapid test for diagnosis of PTB, but its limitation is that its sensitivity is modest in smear negative PTB with or without HIVco-infection in comparison to very high sensitivity in smear-positive PTB. In spite of its modest sensitivity in smear-negative PTB and TB-HIV coinfection, in terms of absolute numbers, CBNAAT adds significantly to the number of microbiologically confirmed PTB in these patients. Main advantage of CBNAAT lies in its Ability in rapid diagnosis and early detection of rifampicin resistance. ${ }^{5}$

Study conducted by D. Pragati Rao et al CBNAAT/RIF can be used as initial test in detecting Tuberculosis in PLHIV, because of its rapidity and detection of Rifampicin resistance. Although sputum should be sent for DST to rule out other drugs resistance.

This ultimately will lead to early treatment and reduced mortality ${ }^{6}$

Study conducted by Giulia Lombardi et al showed that Xpert MTB/RIF is a sensitive method for rapid diagnosis of $\mathrm{TB}$ compared to the conventional $\mathrm{ZN}$ staining. Xpert can serve as a sensitive and time-saving diagnostic method for microbiological diagnosis of smear-negative TB in countries with a low TB prevalence.. ${ }^{7}$
Study conducted by V. Rajkumar et al showed that Significant proportion of patients having Pulmonary tuberculosis with HIV co-infection is smear-negative for acid-fast bacilli. There is a high incidence of tuberculosis among people living with HIV. Sputum microscopy is not a Sensitive diagnostic tool for diagnosing pulmonary tuberculosis in people living with HIV. CBNAAT is a highly sensitive and specific tool for the diagnosis of TB and it is highly Useful for the early diagnosis of smear-negative TB in HIV infected patients.. ${ }^{8}$

Factors affecting sputum smear examination ${ }^{8}$

1-Inadequate smear and improper reading

2-Low bacillary burden especially in females and extremes of age

\section{3-HIV}

\section{4-Lack of cavitory lesion}

However there has been relatively lesser data available with regards to the occurrence of drug resistance in smear negative PTB patints without HIV coinfection. Hence our study estimated the occurence of rifampicin resistance at $8.7 \%$ which is much higher than the regular national incidence of drug resistant PTB (3\% of newly detected PTB as per PMDT 2019).And such patients if not undergoing CB NAAT after being tested negative on smear examination can be the potential source of spread of MDR PTB. hence this mandates the need for compulsory CB NAAT testing even with low index of suspicion.

Limitation of our study were, firstly it was a retrospective study and the associated illnesses like Diabetes mellitus and other conditions were not taken into account which could have been the cause for immunocompromised state leading to sputum smear negativity. Secondly, the clinical 
and radiological correlation with the degree of suspicion was not assessed due to the same reason.

\section{Conclusion}

The study corroborates the potential of CBNAAT in identifying drug resistance in smear negative PTB this should prompt the treating physician to get a CB NAAT testing done even in case of low index of clinical suspicion.

\section{References}

1. HARRISON'S principles of internal medicine 20th edition 173;1236.

2. World Health Organization. Global tuberculosis report Geneva: WHO, 2014. Http://apps.who.int/iris/ bitstream/10665/137094/1/9789 241564809_eng.pdf?Ua=1.

3. Automated real-time nucleic acid amplification technology for rapid and simultaneous detection of tuberculosis and rifampicin resistance: Xpert MTB/RIF system for the diagnosis of pulmonary and extrapulmonary TB in adults and children: policy update. Geneva: World Health Organization, 2013 http://www.who.int/tb/laboratory/policy_st atements/en/)

4. Lawn SD, Nicol MP. Xpert® MTB/RIF assay: development, evaluation and implementation of a new rapid molecular diagnostic for tuberculosis and rifampicin resistance. Future Microbiol 2011;6(9):1067-82

5. J. Evolution Med. Dent. Sci./eissn- 22784802, pissn- 2278-4748/ Vol. 6/ Issue 74/ Sept. 14, 2017

6. J. Evid. Based Med. Healthc., pissn- 23492562, eissn- 2349-2570/ Vol. 3/Issue 38/May 12, 2016

7. PLOS ONE https://doi.org/10.1371/journal.pone.01761 86 April 21, 2017
8. V. Rajkumar, Karthikeyan. A study on early detection of pulmonary tuberculosis in smear negative retroviral positive patients by using CBNAAT. IAIM, 2019; 6(3): 349-354. 